\title{
Article
}

\section{First-Order Formalism and Thick Branes in Mimetic Gravity}

\author{
Qun-Ying Xie ${ }^{1,2}$, Qi-Ming Fu ${ }^{3}$, Tao-Tao Sui ${ }^{2}$, Li Zhao ${ }^{2}$ and Yi Zhong ${ }^{4,5, *}$ \\ 1 School of Information Science and Engineering, Lanzhou University, Lanzhou 730000, China; \\ xieqy@lzu.edu.cn \\ 2 Lanzhou Center for Theoretical Physics \& Research Center of Gravitation, Lanzhou University, \\ Lanzhou 730000, China; suitt14@lzu.edu.cn (T.-T.S.); lizhao@lzu.edu.cn (L.Z.) \\ 3 Institute of Physics, Shaanxi University of Technology, Hanzhong 723000, China; fuqiming@snut.edu.cn \\ 4 School of Physics and Electronics, Hunan University, Changsha 410082, China \\ 5 Hunan Provincial Key Laboratory of High-Energy Scale Physics and Applications, Hunan University, \\ Changsha 410082, China \\ * Correspondence: zhongy@hnu.edu.cn
}

check for updates

Citation: Xie, Q.-Y.; Fu, Q.-M.; Sui, T.-T.; Zhao, L.; Zhong, Y. First-Order Formalism and Thick Branes in Mimetic Gravity. Symmetry 2021, 13, 1345. https://doi.org/10.3390/ sym 13081345

Academic Editors: Ignatios Antoniadis and Kazuharu Bamba

Received: 14 June 2021

Accepted: 22 July 2021

Published: 26 July 2021

Publisher's Note: MDPI stays neutral with regard to jurisdictional claims in published maps and institutional affiliations.

Copyright: (c) 2020 by the authors. Licensee MDPI, Basel, Switzerland. This article is an open access article distributed under the terms and conditions of the Creative Commons Attribution (CC BY) license (https:// creativecommons.org/licenses/by/ $4.0 /)$.

\begin{abstract}
In this paper, we investigate thick branes generated by a scalar field in mimetic gravity theory, which is inspired by considering the conformal symmetry under the conformal transformation of an auxiliary metric. By introducing two auxiliary super-potentials, we transform the second-order field equations of the system into a set of first-order equations. With this first-order formalism, several types of analytical thick brane solutions are obtained. Then, tensor and scalar perturbations are analyzed. We find that both kinds of perturbations are stable. The effective potentials for the tensor and scalar perturbations are dual to each other. The tensor zero mode can be localized on the brane while the scalar zero mode cannot. Thus, the four-dimensional Newtonian potential can be recovered on the brane.
\end{abstract}

Keywords: thick brane; mimetic gravity; first-order formalism

\section{Introduction}

Modified gravity theories have obtained great development and performance in the study of some unsolved problems in general relativity such as the dark energy problem, the dark matter problem, the singularity problem, etc. The symmetry of the theory is crucial in modifying the general relativity. By isolating the conformal degree of freedom of general relativity, Chamseddine and Mukhanov proposed a theory called mimetic gravity [1]. In this theory, an auxiliary metric is introduced, and the physical metric has conformal symmetry under the conformal transformation of an auxiliary metric. This theory was studied from the view of variational principle [2]. It was shown that after introducing the scalar potential, this conformal degree of freedom becomes dynamical and can mimic cold dark matter [1,3] or dark energy [4-7] and can resolve the singularity problem [8] and the cosmic coincidence problem [9]. The extension of mimetic gravity was used to investigate the inflationary solution [10]. In Ref. [11], the authors proposed mimetic Einstein-Cartan gravity and proved that torsion is a non-propagating field in this mimetic gravity. Mimetic gravity theory was also extended to Horava-like theory and applied to galactic rotation curves [12]. It was also applied to other gravity theories such as $f(R)$ gravity [13-19], Horndeski gravity [20-22] and Gauss-Bonnet gravity [23,24].

On the other hand, in order to solve the gauge hierarchy problem and the cosmological constant problem, Randall and Sundrum (RS) proposed that our four-dimensional world could be a brane embedded in five-dimensional space-time [25]. With the warped extra dimension, it was further found that the size of extra dimension can be infinitely large without conflicting with Newtonian gravitational law [26]. This charming idea has attracted substantial researches in particle physics, cosmology, gravity theory, and other related fields [27-37]. In the RS model, the brane is geometrically thin, therefore the space-time 
is singular at the brane. The singularity at a finite distance from the brane is investigated in Refs. [38-41]. Hence, the thick brane model as a generalization of the RS II model is investigated, and various matter fields can be localized on the thick brane [42-48].

Recently, one of the interesting works appeared in Ref. [49], which applied mimetic gravity theory to the thin RSII brane model [26]. It was shown that the mimetic scalar field can mimic the dark sectors on the brane and explain the late time cosmic expansion in the favor of observational data, and it has the capability to explain initial time cosmological inflation [49]. Later, other related topics about thick branes in mimetic gravity were studied in Refs. [50-53]. Thick branes with the inner structure and the stability of the perturbations were first investigated in Ref. [52]. Besides, it is known that first-order formalism is a very powerful tool to obtain analytical brane solutions [54-56]. With this formalism, the secondorder coupled field equations can be written as a set of first-order ones by introducing one or more auxiliary super-potentials. Very recently, Bazeia et al. used first-order formalism to find brane solutions in mimetic gravity [53,57]. In this paper, we would like to investigate thick branes in mimetic gravity with the help of first-order formalism by including two super-potentials. In order to show the systematicness and effectiveness of the first-order formalism on finding analytical brane solutions, we will utilize polynomial, period, and mixed super-potentials. We will also investigate the stabilities of the tensor and scalar perturbations, as well as the relationship between the localizations of the tensor and scalar zero modes.

The paper is organized as follows. In Section 2, we give a review of the thick brane model and reduce the second-order field equations to the first-order ones by introducing two auxiliary super-potentials. In Section 3, we obtain three types of analytical brane solutions by considering different forms of super-potentials. In Sections 4 and 5 , we focus on tensor and scalar perturbations, respectively. Finally, the conclusion and discussion are given in Section 6.

\section{First-Order Formalism for Thick Brane Models}

We consider thick branes generated by a scalar field in five-dimensional mimetic gravity. The corresponding action is given by

$$
S=\int d^{4} x d y \sqrt{-g}\left(\frac{R}{2}+L_{\phi}\right),
$$

where $R$ is the five-dimensional scalar curvature, the Lagrangian of the mimetic scalar field $\phi$ is

$$
L_{\phi}=\lambda\left[g^{M N} \partial_{M} \phi \partial_{N} \phi-U(\phi)\right]-V(\phi) .
$$

Here $\lambda$ represents the Lagrange multiplier, $U(\phi)$ and $V(\phi)$ are two potentials. In this paper, $x^{M}$ and $x^{\mu}$ denote, respectively, the five-dimensional bulk coordinates and the fourdimensional brane ones, where the indices $M, N, \cdots=0,1,2,3,5$ and $\mu, v, \cdots=0,1,2,3$.

The variations of the action (1) with respect to the background metric $g_{M N}$, the scalar field $\phi$, and the Lagrange multiplier $\lambda$ lead to the following field equations

$$
\begin{aligned}
G_{M N}+2 \lambda \partial_{M} \phi \partial_{N} \phi-L_{\phi} g_{M N} & =0, \\
2 \lambda \square^{(5)} \phi+2 \nabla_{M} \lambda \nabla^{M} \phi+\lambda \frac{\partial U}{\partial \phi}+\frac{\partial V}{\partial \phi} & =0, \\
g^{M N} \partial_{M} \phi \partial_{N} \phi-U(\phi) & =0 .
\end{aligned}
$$

Here the five-dimensional d'Alembert operator is defined as $\square^{(5)}=g^{M N} \nabla_{M} \nabla_{N}$. 
The line-element ansatz for a flat brane which preserves the four-dimensional Poincaré symmetry is

$$
\begin{aligned}
d s^{2} & =e^{2 A(y)} \eta_{\mu v} d x^{\mu} d x^{v}+d y^{2} \\
& =a^{2}(y) \eta_{\mu v} d x^{\mu} d x^{v}+d y^{2} .
\end{aligned}
$$

Using the line-element (6), and considering that the scalar field is static and depends only on the extra-dimensional coordinate, we can get the following second-order nonlinear coupled differential field equations

$$
\begin{aligned}
6 A^{\prime 2}+\lambda\left(U(\phi)+\phi^{\prime 2}\right)+V(\phi) & =0 \\
6 A^{\prime 2}+3 A^{\prime \prime}+\lambda\left(U(\phi)-\phi^{\prime 2}\right)+V(\phi) & =0 \\
\lambda\left(8 A^{\prime} \phi^{\prime}+2 \phi^{\prime \prime}+\frac{\partial U}{\partial \phi}\right)+2 \lambda^{\prime} \phi^{\prime}+\frac{\partial V}{\partial \phi} & =0 \\
\phi^{\prime 2}-U(\phi) & =0 .
\end{aligned}
$$

Here, the primes denote derivatives with respect to the extra-dimensional coordinate $y$. It can be seen that it is not easy to analytically solve the above second-order field equations directly. However, one can reduce them to first-order field equations by introducing two auxiliary super-potentials [53]

$$
Q=Q(\phi), \quad W=W(\phi),
$$

and providing the potential

$$
V(\phi)=Q_{\phi} W_{\phi}-\frac{2}{3} W^{2},
$$

where $Q_{\phi}=\frac{d Q}{d \phi}$ and $W_{\phi}=\frac{d W}{d \phi}$. The resulting first-order field equations can be written as

$$
\begin{aligned}
A^{\prime} & =-\frac{1}{3} W(\phi), \\
\phi^{\prime} & =Q_{\phi}, \\
U(\phi) & =Q_{\phi^{\prime}}^{2} \\
\lambda(\phi) & =-\frac{1}{2} \frac{W_{\phi}}{Q_{\phi}} .
\end{aligned}
$$

These equations would be helpful to give analytical brane solutions. One can see from (16) that the scalar potential $U(\phi)$ is related with the super-potential $Q(\phi)$, which is a function of the scalar field $\phi$. The other potential $V(\phi)$ is determined by the two super-potentials $Q$ and $W$ (see Equation (13)).

Note that the first-order Equations (14)-(16) can be divided into two groups. One is Equation (14) related with $A$ and $W$, the other is Equations (15) and (16) related with $\phi, Q, U$. Therefore, these equations can be solved with different approaches by giving different combinations from $(A, W)$ and $(\phi, Q, U)$. For example, we can choose $W(\phi)$ and $Q(\phi)$, or $W(\phi)$ and $\phi(y)$.

The energy density of the brane is given by

$$
\rho(y)=T_{M N} u^{M} u^{N},
$$

where $u^{M}=\left(u^{0}, 0,0,0,0\right)$ is the velocity of a static observer, and the energy momentum tensor of the mimetic scalar field is given by $T_{M N}=2 \lambda \partial_{M} \phi \partial_{N} \phi-L_{\phi} g_{M N}$. From the 
condition of the velocity $g_{M N} u^{M} u^{N}=-1$, we have $u^{0}=\mathrm{e}^{-A}$. Thus, using Equation (11), the energy density can be written as

$$
\rho(y)=\frac{1}{2} V(\phi(y))
$$

which shows that the potential $V(\phi)$ and the profile of the scalar $\phi(y)$ determine the distribution of the thick brane along the extra dimension.

On the other hand, in order to localize gravity on the brane, the warp factor $\mathrm{e}^{2 A(y)}$ should tend to zero rapidly enough as $y \rightarrow \pm \infty$, such that the condition $\int e^{2 A} d y<\infty$ is satisfied. This will be derived in Section 4 . Usually, we consider the solutions with $\left.e^{2 A(y)}\right|_{y \rightarrow \pm \infty} \rightarrow e^{-2 k|y|}$, which corresponds to the branes embedded in an AdS spacetime with a negative cosmological constant. It should be pointed out that the contribution of the cosmological constant has been included in the energy density (19) for this case. Therefore, the net energy density should be

$$
\rho(y)=\frac{1}{2} V(\phi(y))-\Lambda .
$$

\section{The Thick Brane Solutions}

Next, we will take focus on finding some specific analytical solutions of the thick brane model by solving the first-order Equations (14)-(17). Our main motivation here is to show the systematization and effectiveness of the first-order formalism, which is also called the super-potential method $[34,35,58]$. In the symmetric thick brane scenario,the warped factor $a(y)$ is an odd function. Furthermore, the scalar field is a kink that connected two vacuum, Combining with Equations (13)-(15), $W(\phi(y))$ must be an odd function of $y$, $Q_{\phi}(\phi(y))$ must be an even function of $y$, and the potential $V(\phi)$ must have a multi-vacuum. In order to be compatible with the above analysis, we consider the polynomial and period super-potentials.

\subsection{Polynomial Super-Potentials}

\subsubsection{Solution I}

First, supposing that one of the super-potentials, $Q$, has the following polynomial form considered in Ref. [53]:

$$
Q(\phi)=k\left(v \phi-\frac{\phi^{3}}{3 v}\right)
$$

and solving Equation (15), we can easily get the solution of the scalar field $\phi$

$$
\phi(y)=v \tanh (k y) .
$$

The solution of the potential $U$ can be read directly from Equation (16) as

$$
U(\phi)=\frac{k^{2}}{v^{2}}\left(\phi^{2}-v^{2}\right)^{2} .
$$

The other super-potential, $W$, is chosen as

$$
W(\phi)=\frac{3 k n}{v} \phi,
$$

where $n$ is a non-vanishing parameter. Then, from Equation (14), we obtain a simple solution for the warp factor

$$
A(y)=\ln \operatorname{sech}^{n}(k y) .
$$


The other functions are given by

$$
\begin{aligned}
\lambda(y) & =-\frac{3 n}{2 v^{2}} \cosh ^{2}(k y), \\
V(\phi) & =\frac{3 n k^{2}}{v^{2}}\left[v^{2}-(2 n+1) \phi^{2}\right] .
\end{aligned}
$$

The energy density of the brane including the cosmological constant is

$$
\rho^{(\Lambda)}=\frac{V}{2}=3 k^{2} n\left(1-(2 n+1) \tanh ^{2}(k y)\right) .
$$

From the solution (25), we can calculate the cosmological constant and hence the net energy density

$$
\rho(y)=\rho^{(\Lambda)}-\Lambda=\frac{3}{2} k^{2} n(2 n+1) \operatorname{sech}^{2}(k y) .
$$

The shapes of the warp factor $a(y)=\mathrm{e}^{A(y)}$ and the energy density $\rho(y)$ are plotted in Figure 1, which shows that the parameter $n$ affects the warp factor and the energy density. With the increase of $n$, the warp factor becomes narrower while the energy density becomes larger and narrower. The maximum of the energy density is given by $\rho_{\max }=\frac{3}{2} k^{2} n(2 n+1)$ for $n>0$ or $n<-1 / 2$. It is obvious that the parameter $v$ does not affect the warp factor and the energy density, it only affects the amplitude of the scalar field $\phi$ and hence the localization of a bulk fermion $\Psi$ when one introduces the Yukawa coupling $\eta \bar{\Psi} \phi \Psi$ [59]. In fact, $v$ is the vacuum expectation value of the scalar potential $U(\phi)$ given in (23).
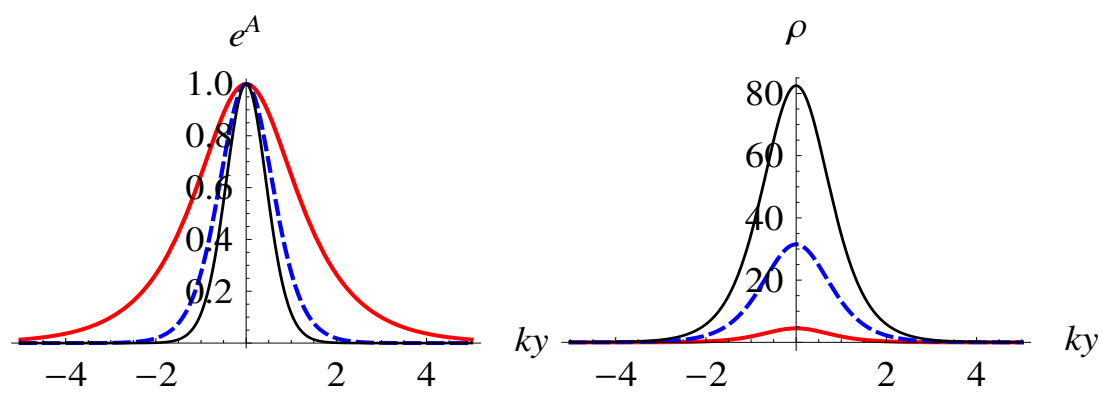

Figure 1. The shapes of the warp factor $a(y)$ and the energy density $\rho(y)$ of the first brane model. The parameter $n$ is set as $n=1,3,5$ for the thick red, dashed blue, and thin black lines, respectively.

\subsubsection{Solution II}

Next, we also consider the same polynomial super-potential $Q$ as in previous subsection:

$$
Q(\phi)=k\left(v \phi-\frac{\phi^{3}}{3 v}\right) .
$$

Therefore, we will get the same $\phi$ and $U(\phi)$ as solution I:

$$
\begin{aligned}
\phi(y) & =v \tanh (k y), \\
U(\phi) & =\frac{k^{2}}{v^{2}}\left(\phi^{2}-v^{2}\right)^{2} .
\end{aligned}
$$

However, different from last model, we fix $W(\phi)=Q(\phi)$, which results in the constant solution of the Lagrange multiplier:

$$
\lambda(y)=-\frac{1}{2} \frac{W_{\phi}}{Q_{\phi}}=-\frac{1}{2} .
$$


Then, we get a different form of the warp factor via Equation (14):

$$
A(y)=\frac{v^{2}}{18} \operatorname{sech}^{2}(k y)+\frac{2 v^{2}}{9} \ln \operatorname{sech}(k y) .
$$

The scalar potential $V$ has the following $\phi^{6}$ form:

$$
V(\phi)=\frac{k^{2}}{v^{2}}\left(\phi^{2}-v^{2}\right)^{2}-\frac{2 k^{2}}{9 v^{2}}\left(\phi^{2}-3 v^{2}\right)^{2} \phi^{2} .
$$

In this case, the energy density of the brane is

$$
\begin{aligned}
\rho(y) & =\frac{k^{2} v^{2}}{108}\left(27+10 v^{2}+3\left(9+2 v^{2}\right) \cosh (2 k y)\right) \\
& \times \operatorname{sech}^{6}(k y) .
\end{aligned}
$$

In this model, the vacuum expectation value $v$ of the scalar potential $U(\phi)$ has an explicit effect on the warp factor and the energy density. The shapes of the scalar field, the warp factor, and the energy density do not change with non-vanishing $v$. Note that

$$
\begin{aligned}
A(|y| \rightarrow \infty) & \rightarrow \frac{2}{9} v^{2}\left(e^{-2 k|y|}-k|y|\right) \\
& \rightarrow-\frac{2}{9} v^{2} k|y| .
\end{aligned}
$$

Therefore, the five-dimensional spacetime is also asymptotic AdS.

\subsection{Period Super-Potential}

\subsubsection{Solution III}

Next, we try to construct another form of brane solution by giving a period superpotential, such as

$$
W(\phi)=3 k n \sin ^{\frac{1}{p}}\left(\frac{\phi}{v}\right) .
$$

At the same time, the warp factor $A(y)$ is assumed to be

$$
A(y)=\ln \operatorname{sech}^{n}(k y) .
$$

Then, the scalar field can be solved from Equation (14) as

$$
\phi(y)=v s \cdot \arcsin \left[\tanh ^{p}(k y)\right],
$$

which is a kink and a double kink for $p=1$ and $p=2 n+1$ with positive integer $n$, respectively. Furthermore, from Equation (15) we know that the super-potential $Q$ is

$$
Q(\phi)=k p^{2} v^{2}[\mathcal{F}(p, \phi)+\mathcal{F}(-p, \phi)] .
$$

where

$$
\begin{aligned}
\mathcal{F}(p, \phi) & =(-1)^{\frac{1}{2 p}} \sec ^{\frac{1}{p}}\left(\frac{\phi}{v}\right) \\
& \times{ }_{2} F_{1}\left(\frac{1}{2 p}, \frac{1}{2 p} ; 1+\frac{1}{2 p} ; \sec ^{2}\left(\frac{\phi}{v}\right)\right)
\end{aligned}
$$


Here, ${ }_{2} F_{1}$ is the hypergeometric function. The Lagrange multiplier and the two potentials can also be solved:

$$
\begin{aligned}
\lambda(y) & =\frac{3 n \cot ^{2}\left(\frac{\phi}{v}\right) \sin ^{\frac{2}{p}}\left(\frac{\phi}{v}\right)}{2 p^{2} v^{2}\left[\sin ^{\frac{2}{p}}\left(\frac{\phi}{v}\right)-1\right]} \\
V(\phi) & =3 k^{2} n\left[1-(1+2 n) \sin ^{\frac{2}{p}}\left(\frac{\phi}{v}\right)\right], \\
U(\phi) & =k^{2} p^{2} v^{2} \operatorname{sech}^{2}\left(\frac{\phi}{v}\right) \\
& \times\left[\sin ^{1+\frac{1}{p}}\left(\frac{\phi}{v}\right)-\sin ^{1-\frac{1}{p}}\left(\frac{\phi}{v}\right)\right]^{2} .
\end{aligned}
$$

The energy density in this case is given by

$$
\rho(y)=\frac{3}{2} k^{2} n(2 n+1) \operatorname{sech}^{2}(k y),
$$

which is the same as Equation (29) for the first model. In fact, from the definition of the energy density $\rho=T_{M N} u^{M} u^{N}=(1 / 2) G_{M N} u^{M} u^{N}$, we know that the energy density will have the same configuration for the same warp factor. Here, the warp factors in this model and the first model have the same form, and hence so do the energy densities.

\subsubsection{Solution IV}

Similarly, for the period super-potentials

$$
Q(\phi)=W(\phi)=k v^{2} \sin \left(\frac{\phi}{v}\right)
$$

we can obtain the following solution

$$
\begin{aligned}
A(y) & =\frac{v^{2}}{3} \ln [\operatorname{sech}(k y)] \\
\phi(y) & =v \operatorname{sirctan}[\sinh (k y)] \\
\lambda(y) & =-\frac{1}{2} \\
V(\phi) & =k^{2} v^{2}\left(\cos ^{2}\left(\frac{\phi}{v}\right)-\frac{2}{3} v^{2} \sin ^{2}\left(\frac{\phi}{v}\right)\right), \\
U(\phi) & =k^{2} v^{2} \cos ^{2} \frac{\phi}{v} \\
\rho(y) & =\frac{1}{6} k^{2} v^{2}\left(2 v^{2}+3\right) \operatorname{sech}^{2}(k y) .
\end{aligned}
$$

\subsubsection{Solution $\mathrm{V}$}

Next, we consider different period super-potentials $W(\phi)$ and $Q(\phi)$ :

$$
\begin{aligned}
W(\phi) & =3 k n \tan \left(\frac{\phi}{v}\right), \\
Q(\phi) & =\frac{1}{2} k v^{2} \sin \left(\frac{2 \phi}{v}\right),
\end{aligned}
$$

The warp factor and the scalar field will have the following explicit forms

$$
\begin{aligned}
A(y) & =\ln \left[\operatorname{sech}^{n}(k y)\right], \\
\phi(y) & =v s \cdot \arctan [\tanh (k y)] .
\end{aligned}
$$


The other functions are given by

$$
\begin{aligned}
\lambda(y) & =-\frac{3 n}{2 v^{2}} \sec [2 \arctan \tanh (k y)], \\
& \times\left(1+\tanh ^{2}(k y)\right), \\
V(\phi) & =3 k^{2} n \sec ^{2}\left(\frac{\phi}{v}\right)\left[(1+n) \cos \left(\frac{2 \phi}{v}\right)-n\right], \\
U(\phi) & =k^{2} v^{2} \cos ^{2}\left(\frac{2 \phi}{v}\right) .
\end{aligned}
$$

\subsection{Mixed Super-Potential}

\subsubsection{Solution VI}

Finally, we would like to generate the brane model by giving the pair of superpotentials $Q(\phi)$ and $W(\phi)$ as the mixed of polynomial and period, for example

$$
Q(\phi)=W(\phi)=k v\left[\phi+v s \cdot \sin \left(\frac{\phi}{v}\right)\right],
$$

which results in the constant Lagrange multiplier

$$
\lambda(y)=-\frac{1}{2} .
$$

Then, governed by Equation (15), the scalar field $\phi$ is determined as

$$
\phi(y)=2 v \arctan (k y),
$$

from which one can see that the asymptotic behavior of $\phi$ is $\lim _{y \rightarrow \pm \infty} \phi(y)=\pi$. The potentials $U$ and $V$ are

$$
\begin{aligned}
& U(\phi)=4 k^{2} v^{2} \cos ^{4}\left(\frac{\phi}{2 v}\right), \\
& V(\phi)=k^{2} v^{2}\left[4 \cos ^{4}\left(\frac{\phi}{2 v}\right)-\frac{2}{3}\left(\phi+v \sin \left(\frac{\phi}{v}\right)\right)^{2}\right] .
\end{aligned}
$$

The warp factor and the energy density read as

$$
\begin{aligned}
A(y) & =-\frac{2}{3} v^{2} k y \arctan (k y), \\
\rho(y) & =\frac{1}{3} k^{2} v^{2}\left[\frac{6}{\left(k^{2} y^{2}+1\right)^{2}}+\pi^{2} v^{2}\right. \\
& \left.-v^{2}(2 \arctan (k y)+\sin (2 \arctan (k y)))^{2}\right] .
\end{aligned}
$$

The asymptotic behavior of $A(y)$ is $A\left(|y| \rightarrow \pm \infty \rightarrow-\pi v^{2} k|y| / 3\right.$.

\section{Tensor Perturbation}

In this section, we consider the linear tensor fluctuation of the metric around the background. Following the previous research works in Refs. [33,52,53], we perform the following coordinate transformation

$$
d z=e^{-A(y)} d y
$$

to get a conformally flat metric

$$
d s^{2}=e^{2 A(z)}\left(\eta_{\mu v} d x^{\mu} d x^{\nu}+d z^{2}\right) .
$$


To simplify the fluctuations of the metric around the background, we only consider the transverse and traceless part of the metric fluctuation, i.e., we consider the following tensor perturbation of the metric:

$$
\begin{aligned}
d s^{2} & =\left(e^{2 A(z)} \eta_{\mu v}+\hat{h}_{\mu v}(x, z)\right) d x^{\mu} d x^{v}+e^{2 A(z)} d z^{2} \\
& =e^{2 A(z)}\left[\left(\eta_{\mu \nu}+h_{\mu v}(x, z)\right) d x^{\mu} d x^{v}+d z^{2}\right] .
\end{aligned}
$$

Here, $h_{\mu v}$ is the tensor perturbation of the metric, and it satisfies the transverse and traceless conditions [34]: $h_{\mu}^{\mu}=\partial^{v} h_{\mu v}=0$. The non-vanishing part of the perturbation of Einstein tensor in Equation (3) is the $\mu \nu$ components (since $h_{55}=0$ and $\delta G_{55}=0$ ) and it reads

$$
\begin{aligned}
\delta G_{\mu v} & =-\frac{1}{2} \square^{(4)} h_{\mu v}+\left(6 A^{\prime 2}+3 A^{\prime \prime}\right) e^{2 A} h_{\mu v} \\
& -2 A^{\prime} e^{2 A} h_{\mu v}^{\prime}-\frac{1}{2} e^{2 A} h_{\mu \nu}^{\prime \prime}
\end{aligned}
$$

where the four-dimensional d'Alembertian is defined as $\square^{(4)} \equiv \eta^{\mu v} \partial_{\mu} \partial_{\nu}$. Using Equation (10), we get the perturbation equation

$$
-\frac{1}{2} \square^{(4)} h_{\mu v}-2 A^{\prime} e^{2 A} h_{\mu \nu}^{\prime}-\frac{1}{2} e^{2 A} h_{\mu \nu}^{\prime \prime}=0 .
$$

Considering Equation (68), we rewrite the above equation under the coordinate $z$ as

$$
\square^{(4)} h_{\mu v}+3 \dot{A} \dot{h}_{\mu v}+\ddot{h}_{\mu v}=0,
$$

where the dot represents the derivative with respect to the coordinate $z$. By performing the following decomposition,

$$
h_{\mu v}(x, z)=\varepsilon_{\mu \nu} e^{i k x} h(z) e^{-\frac{3}{2} A},
$$

Equation (73) leads to a kind of Schrödinger equation:

$$
\left(-\partial_{z}^{2}+V_{\mathrm{T}}(z)\right) h(z)=m^{2} h(z)
$$

where $k^{2}=-m^{2}$ with $m$ the four-dimensional mass of a graviton KK mode, and the effective potential given by

$$
V_{\mathrm{T}}(z)=\frac{3}{2} \ddot{A}(z)+\frac{9}{4} \dot{A}^{2}(z) .
$$

The effective potential of the tensor perturbation under the physical coordinate $y$ is

$$
V_{\mathrm{T}}(z(y))=e^{2 A(y)}\left(\frac{3}{2} A^{\prime \prime}(y)+\frac{15}{4} A^{\prime 2}(y)\right) .
$$

The zero mode of the tensor perturbation reads as

$$
h_{0}(z)=e^{3 A(z) / 2}\left(c_{1}+c_{2} \int e^{-3 A(z)} d z\right) .
$$

This general form of the zero mode was first found in $f(R)$-brane model in Ref. [60]. One can see that the effective potentials and the zero mode of the tensor perturbation are only determined by the warp factor $A(y)$. It is easy to show that the tensor zero mode (78) can be localized on the brane with the choice of $c_{2}=0$ for all brane solutions in this paper (with $n>0$ for solutions I, III and V and $v \neq 0$ for solutions II, IV and VI). 
The figures for solutions I, III and V are the same, and they are similar for solutions II, IV and VI. So, without loss of generality, we plot two of them for the brane solutions I and II in Figures 2 and 3, respectively. These potentials have a volcano-like shape. As the parameters $n$ and $v$ increase, the potential wells in Figures 2 and 3 become narrower and deeper, respectively.
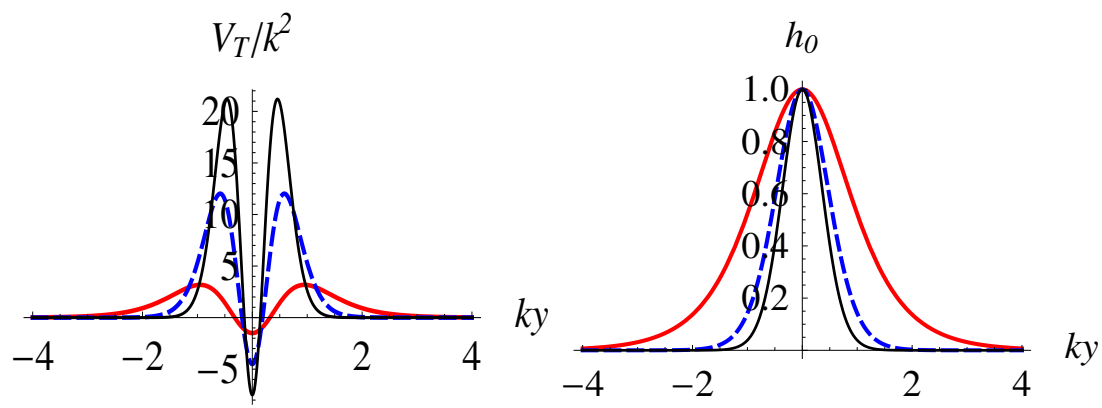

Figure 2. The effective potential $V_{\mathrm{T}}$ and the non-normalized zero mode of the tensor perturbation for brane models I, III and V. The parameter is set as $n=1$ (red solid thick lines), $n=3$ (blue dashed lines), and $n=5$ (black solid thin lines).
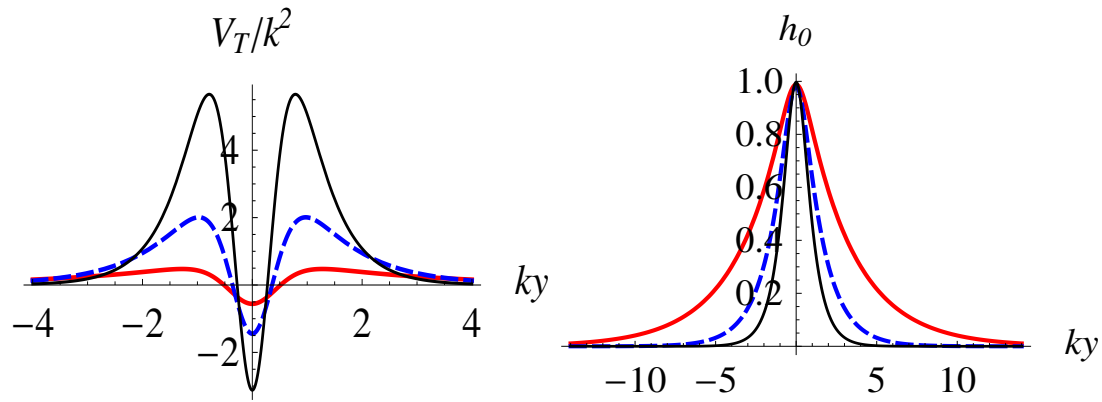

Figure 3. The effective potential $V_{\mathrm{T}}$ and the non-normalized zero mode of the tensor perturbation for brane model II. The parameter is set as $v=1$ (red solid thick lines), $v=1.5$ (blue dashed lines), and $v=2$ (black solid thin lines).

It is easy to verify that the zero modes for the above brane models are localized around the brane. So the four-dimensional Newtonian potential can be realized on the brane. There is no tensor tachyon mode, thus the brane is stable against the tensor perturbation.

\section{Scalar Perturbation}

At last, we come to the scalar perturbation in this section. The perturbed metric is given by

$$
d s^{2}=e^{2 A(z)}\left[(1+2 \psi) \eta_{\mu \nu} d x^{\mu} d x^{\nu}+(1+2 \Phi) d z^{2}\right] .
$$

The scalar perturbation equations can be derived as

$$
\begin{gathered}
e^{2 A}\left(\frac{1}{2} U_{\phi \phi}-U_{\phi} \frac{\ddot{\phi}}{\dot{\phi}^{2}}+2 \frac{\dot{A}}{\dot{\phi}} U_{\phi}-\frac{4}{3} e^{-2 A} \lambda \dot{\phi}^{2}\right) \delta \phi \\
+\left(\frac{1}{2} \frac{e^{2 A}}{\dot{\phi}} U_{\phi}+\frac{\ddot{\phi}}{\dot{\phi}}-2 \dot{A}\right) \dot{\delta \phi}-\ddot{\delta \phi}=0,
\end{gathered}
$$

and

$$
\begin{aligned}
& \Phi=\frac{\dot{\delta \phi}}{\dot{\phi}}-\frac{e^{2 A}}{2 \dot{\phi}^{2}} U_{\phi} \delta \phi, \\
& \Phi=-2 \psi .
\end{aligned}
$$


It can be seen that there is only one degree of freedom for the scalar perturbation. Finally, by using the background Equations (3)-(5), we can replace the potential $U$ and $\lambda$ in Equation (80) with the functions $A$ and $\phi$ and obtain the final form of the scalar perturbation $\delta \phi$ :

$$
\begin{aligned}
& \left(3 \dot{A} \frac{\ddot{\phi}}{\dot{\phi}}-3 \ddot{A}-2 \frac{\ddot{\phi}^{2}}{\dot{\phi}^{2}}+\frac{\dddot{\phi}}{\dot{\phi}}\right) \delta \phi \\
& +\left(2 \frac{\ddot{\phi}}{\dot{\phi}}-3 \dot{A}\right) \dot{\delta \phi}-\ddot{\delta \phi}=0 .
\end{aligned}
$$

Redefining

$$
\delta \phi\left(x^{\mu}, z\right)=\overline{\delta \phi}\left(x^{\mu}\right) s(z) A^{-3 / 2}(z) \dot{\phi}(z),
$$

with the four-dimensional part of $\delta \phi$ satisfying $\square^{(4)} \overline{\delta \phi}\left(x^{\mu}\right)=0$, we get the perturbation equation for the scalar degree of freedom $s(z)$ from Equation (83):

$$
\left(-\partial_{z}^{2}+V_{S}(z)\right) s(z)=0
$$

where the effective potential is given by

$$
V_{\mathrm{S}}(z)=-\frac{3}{2} \ddot{A}(z)+\frac{9}{4} \dot{A}^{2}(z)
$$

in the $z$ coordinate or

$$
V_{\mathrm{S}}(z(y))=-e^{2 A}\left(\frac{3}{2} A^{\prime \prime}(y)+\frac{3}{4} A^{\prime 2}(y)\right)
$$

in the $y$ coordinate. Note that the corresponding effective potential of (86) in Ref. [52] is not right since there is an error in the matter Equation (45) in that paper (the term $+2 \lambda\left(\partial_{z} \phi\right)^{2}$ should be $\left.-2 \lambda\left(\partial_{z} \phi\right)^{2}\right)$.

Comparing (86) with (76), one can see that the effective potential of the scalar perturbation $V_{\mathrm{S}}$ is dual to that of the tensor perturbation $V_{\mathrm{T}}: V_{\mathrm{S}}=V_{\mathrm{T}}(A \rightarrow-A)$, and Equations (85) and (75) can be rewritten as

$$
\begin{aligned}
& \mathcal{P}^{\dagger} \mathcal{P}_{S}(z)=0, \\
& \mathcal{P} \mathcal{P}^{\dagger} h(z)=0,
\end{aligned}
$$

where $\mathcal{P}=\partial_{z}+\frac{3}{2} \dot{A}$. The above two equations ensure that both the scalar and tensor perturbations are stable. The zero mode can be obtained by replacing $A \rightarrow-A$ from the solution of the tensor zero mode (78):

$$
s(z)=e^{-3 A(z) / 2}\left(c_{1}+c_{2} \int e^{3 A(z)} d z\right) .
$$

This will lead to the conclusion that only one of the tensor and scalar zero modes can be localized on the brane.

The effective potential (87) for solution I is given by

$$
V_{\mathrm{S}}=\frac{3}{8} k^{2} n \operatorname{sech}^{2}(k y)(4+n-n \cosh (2 k y)),
$$

which is shown in Figure 4. From this figure, it can be seen that, the value of the effective potential at $z=0$ will increase with the parameter $n$. This can be checked from the expression $V_{\mathrm{S}}(0)=\frac{3 k^{2} n}{2}$. The potential has two very shallow wells, and approaches $0^{-}$ when $y \rightarrow \pm \infty$. 


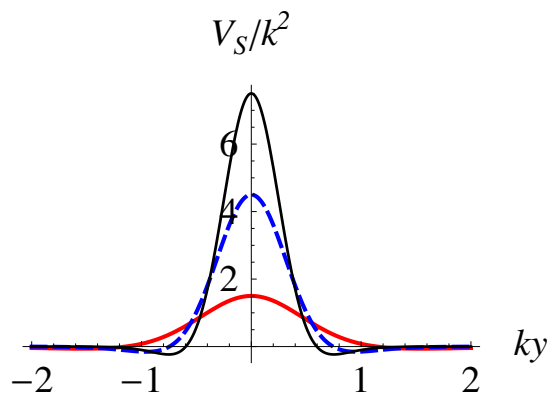

Figure 4. The effective potential $V_{S}(z(y))$ for solution I. The parameter is set as $n=1$ (red solid thick lines), $n=3$ (blue dashed lines), and $n=5$ (black solid thin lines).

For the brane solution I in (25) with $n>0$, it is easy to show that the zero mode (90) of the scalar perturbation cannot be localized on the brane. Thus, there is no additional fifth force coming from the scalar perturbation. For other brane solutions, we have also the same conclusion.

\section{Conclusions}

In this work, we investigated the super-potential method with which the second-order equations can be reduced to the first-order ones for thick brane models in modified gravity with Lagrange multiplier. The main step of this method is to introduce a pair of auxiliary super-potentials, i.e., $W(\phi)$ and $Q(\phi)$. With these two super-potentials, the field equations are rewritten as Equations (13)-(17). Then we try to use the method to find a series of analytical brane solutions via some polynomial super-potentials, period super-potentials, and mixed super-potentials. The warp factor has the same shape and the same asymptotic behavior at the boundary of the extra dimension for all those solutions, and all of these branes are embedded in five-dimensional AdS spacetime. The scalar field $\phi$ is a double kink for (40) with odd integer $p \geq 3$ or a single kink for other solutions. These shapes of the scalar field will affect the localization properties of fermions on the brane through the Yukawa coupling $\eta \bar{\Psi} \phi \Psi$ [59].

We also considered the tensor and scalar perturbations of the brane system. It was shown that both equations of motion of the perturbations can be transformed into Schrödinger-like equations. Furthermore, these equations can be recast as the forms of (88) and (89), which show that both the perturbations are stable. The effective potential of the tensor perturbation is dual to that of the scalar perturbation. Therefore, only one of the tensor and scalar zero modes can be localized on the brane. For all of our brane solutions, the tensor zero mode can be localized on the brane while the scalar zero mode cannot. Thus, the four-dimensional Newtonian potential can be recovered on the brane and there is no additional fifth force contradicting with the experiments.

Author Contributions: Conceptualization, Q.-Y.X.; Q.-M.F.; T.-T.S.; L.Z. and Y.Z.; methodology, Q.-Y.X.; Q.-M.F.; T.-T.S.; L.Z. and Y.Z.; writing-original draft preparation, Q.-Y.X.; Q.-M.F.; T.-T.S.; L.Z. and Y.Z.; writing-review and editing, Q.-Y.X.; Q.-M.F.; T.-T.S.; L.Z. and Y.Z. All authors have read and agreed to the published version of the manuscript.

Funding: Thiswork was supported by the National Natural Science Foundation of China (Grant No. 11875151, No. 12047501, and No. 11705070). Yi Zhong was supported by the Fundamental Research Funds for the Central Universities (Grant No. 531107051196).

Conflicts of Interest: The authors declare no conflict of interest. 


\section{References}

1. Chamseddine, A.H.; Mukhanov, V. Mimetic Dark Matter. J. High Energy Phys. 2013, 2013, 135. [CrossRef]

2. Golovnev, A. On the recently proposed Mimetic Dark Matter. Phys. Lett. 2014, 728, 39-40. [CrossRef]

3. Chamseddine, A.H.; Mukhanov, V.; Vikman, A. Cosmology with Mimetic Matter. J. Cosmol. Astropart. Phys. 2014, $2014,017$. [CrossRef]

4. Casalino, A.; Rinaldi, M.; Sebastiani, L.; Vagnozzi, S. Mimicking dark matter and dark energy in a mimetic model compatible with GW170817. Phys. Dark Univ. 2018, 22, 108. [CrossRef]

5. Matsumoto, J. Unified description of dark energy and dark matter in mimetic matter model. arXiv 2016, arXiv:1610.07847.

6. Nojiri, S.; Odintsov, S.D.; Oikonomou, V.K. Unimodular-Mimetic Cosmology. Class. Quant. Grav. 2016, 33, 125017. [CrossRef]

7. Nojiri, S.; Odintsov, S.D.; Oikonomou, V.K. Viable Mimetic Completion of Unified Inflation-Dark Energy Evolution in Modified Gravity. Phys. Rev. 2016, 94, 104050. [CrossRef]

8. Brahma, S.; Golovnev, A.; Yeoma, D.H. On singularity-resolution in mimetic gravity. Phys. Lett. 2018, 782, 280. [CrossRef]

9. Dutta, J.; Khyllep, W.; Saridakis, E.N.; Tamanini, N.; Vagnozzi, S. Cosmological dynamics of mimetic gravity. J. Cosmol. Astropart. Phys. 2018, 1802, 041. [CrossRef]

10. Mansoori, S.A.H.; Talebian, A.; Firouzjahi, H. Mimetic Inflation. arXiv 2010, arXiv:2010.13495.

11. Izaurieta, F.; Medina, P.; Merino, N.; Salgado, P.; Valdivia, O. Mimetic Einstein-Cartan-Kibble-Sciama (ECKS) gravity. arXiv 2020, arXiv:2007.07226.

12. Sebastiani, L.; Vagnozzi, S.; Myrzakulov, R. Mimetic gravity: A review of recent developments and applications to cosmology and astrophysics. Adv. High Energy Phys. 2017, 2017, 3156915. [CrossRef]

13. Nozari, K.; Sadeghnezhad, N. Braneworld mimetic f(R) gravity. Int. J. Geom. Meth. Mod. Phys. 2019, 16, 1950042. [CrossRef]

14. Momeni, D.; Myrzakulov, R.; Güdekli, E. Cosmological viable mimetic $f(R)$ and $f(R, T)$ theories via Noether symmetry. Int. J. Geom. Meth. Mod. Phys. 2015, 12, 1550101. [CrossRef]

15. Nojiri, S.; Odintsov, S.D. Mimetic f(R) gravity: Inflation, dark energy and bounce. Mod. Phys. Lett. A 2014, 29, 1450211. [CrossRef]

16. Odintsov, S.D.; Oikonomou, V.K. Mimetic $F(R)$ inflation confronted with Planck and BICEP2/Keck Array data. Astrophys. Space Sci. 2016, 361, 174. [CrossRef]

17. Odintsov, S.D.; Oikonomou, V.K. Viable Mimetic $F(R)$ Gravity Compatible with Planck Observations. Ann. Phys. 2015, 363, 503-514. [CrossRef]

18. Odintsov, S.D.; Oikonomou, V.K. Accelerating cosmologies and the phase structure of F(R) gravity with Lagrange multiplier constraints: A mimetic approach. Phys. Rev. 2016, 93, 023517. [CrossRef]

19. Oikonomou, V.K. Reissner-Nordström Anti-de Sitter Black Holes in Mimetic $F(R)$ Gravity. Universe 2016, 2, 10. [CrossRef]

20. Arroja, F.; Okumura, T.; Bartolo, N.; Karmakar, P.; Matarrese, S. Large-scale structure in mimetic Horndeski gravity. J. Cosmol. Astropart. Phys. 2018, 2018, 050. [CrossRef]

21. Arroja, F.; Bartolo, N.; Karmakar, P.; Matarrese, S. Cosmological perturbations in mimetic Horndeski gravity. J. Cosmol. Astropart. Phys. 2016, 2016, 042. [CrossRef]

22. Cognola, G.; Myrzakulov, R.; Sebastiani, L.; Vagnozzi, S.; Zerbini, S. Covariant Hořava-like and mimetic Horndeski gravity: Cosmological solutions and perturbations. Class. Quant. Grav. 2016, 33, 225014. [CrossRef]

23. Capozziello, S.; Makarenko, A.N.; Odintsov, S.D. Gauss-Bonnet dark energy by Lagrange multipliers. Phys. Rev. 2013, 87, 084037. [CrossRef]

24. Astashenok, A.V.; Odintsov, S.D.; Oikonomou, V.K. Modified Gauss-Bonnet gravity with the Lagrange multiplier constraint as mimetic theory. Class. Quant. Grav. 2015, 32, 185007. [CrossRef]

25. Randall, L.; Sundrum, R. A Large mass hierarchy from a small extra dimension. Phys. Rev. Lett. 1999, 83, 3370-3373. [CrossRef]

26. Randall, L.; Sundrum, R. An Alternative to compactification. Phys. Rev. Lett. 1999, 83, 4690-4693. [CrossRef]

27. Davoudiasl, H.; Hewett, J.L.; Rizzo, T.G. Bulk gauge fields in the Randall-Sundrum model. Phys. Lett. 2000, 473, 43-49. [CrossRef]

28. Shiromizu, T.; Maeda, K.I.; Sasaki, M. The Einstein equation on the 3-brane world. Phys. Rev. 2000, 62, 024012. [CrossRef]

29. Gherghetta, T.; Pomarol, A. Bulk fields and supersymmetry in a slice of AdS. Nucl. Phys. 2000, 586, 141-162. [CrossRef]

30. Rizzo, T.G. Introduction to Extra Dimensions. AIP Conf. Proc. 2010, 1256, 27-50. [CrossRef]

31. Yang, K.; Liu, Y.-X.; Zhong, Y.; Du, X.-L.; Wei, S.-W. Gravity localization and mass hierarchy in scalar-tensor branes. Phys. Rev. 2012, 86, 127502. [CrossRef]

32. Agashe, K.; Azatov, A.; Cui, Y.; Randall, L.; Son, M. Warped Dipole Completed, with a Tower of Higgs Bosons. J. High Energy Phys. 2015, 2015, 196. [CrossRef]

33. Csaki, C.; Erlich, J.; Hollowood, T.J.; Shirman, Y. Universal aspects of gravity localized on thick branes. Nucl. Phys. 2000, 581, 309-338. [CrossRef]

34. DeWolfe, O.; Freedman, D.Z.; Gubser, S.S.; Karch, A. Modeling the fifth-dimension with scalars and gravity. Phys. Rev. 2000, 62, 046008. [CrossRef]

35. Gremm, M. Four-dimensional gravity on a thick domain wall. Phys. Lett. 2000, 478, 434-438. [CrossRef]

36. Liu, Y.-X. Introduction to Extra Dimensions and Thick Braneworlds. arXiv 2017, arXiv:1707.08541.

37. Rosa, J.L.; Ferreira, D.A.; Bazeia, D.; Lobo, F.S.N. Thick brane structures in generalized hybrid metric-Palatini gravity. Eur. Phys. J. 2021, 81, 20. [CrossRef]

38. Antoniadis, I.; Cotsakis, S.; Klaoudatou, I. Brane singularities with a scalar field bulk. arXiv 2010, arXiv:1004.3379. 
39. Antoniadis, I.; Cotsakis, S.; Klaoudatou, I. Brane singularities and their avoidance. Class. Quant. Grav. 2010, 27, 235018. [CrossRef]

40. Antoniadis, I.; Cotsakis, S.; Klaoudatou, I. Brane singularities and their avoidance in a fluid bulk. arXiv 2010, arXiv:1005.3221.

41. Antoniadis, I.; Cotsakis, S.; Klaoudatou, I. Enveloping branes and brane-world singularities. Eur. Phys. J. C 2014, 74, 3192. [CrossRef]

42. Arai, M.; Blaschke, F.; Eto, M.; Sakai, N. Grand Unified Brane World Scenario. arXiv 2017, arXiv:1705.02839.

43. Chinaglia, M.; Cruz, W.T.; Correa, R.A.C.; de Paula, W.; Moraes, P.H.R.S. Configurational Entropy as a tool to select a physical Thick Brane Model. arXiv 2017, arXiv:1710.04518.

44. Bazeia, D.; Lima, E.E.M.; Losano, L. Kinks and branes in models with hyperbolic interactions. Int. J. Mod. Phys. 2017, $32,1750163$. [CrossRef]

45. Menezes, R.; Moreira, D.C. New models for asymmetric kinks and branes. Ann. Phys. 2017, 383, 662. [CrossRef]

46. Gogberashvili, M.; Midodashvili, P. Diffractions from the brane and GW150914. Europhys. Lett. 2015, 114, 50008. [CrossRef]

47. Bazeia, D.; Marques, M.A.; Menezes, R. Models for asymmetric hybrid brane. Phys. Rev. 2015, 92, 084058. [CrossRef]

48. Kastor, D.; Ray, S.; Traschen, J. Lovelock Branes. arXiv 2017, arXiv:1706.06684.

49. Sadeghnezhad, N.; Nozari, K. Braneworld Mimetic Cosmology. Phys. Lett. 2017, 769, 134-140. [CrossRef]

50. Zhong, Y.; Zhong, Y.; Zhang, Y.-P.; Guo, W.-D.; Liu, Y.-X. Gravitational resonances in mimetic thick branes. arXiv 2019, arXiv:1812.06453.

51. Xiang, Q.; Zhong, Y.; Xie, Q.-Y.; Zhao, L. Flat and bent branes with inner structure in two-field mimetic gravity. arXiv, arXiv:2011.10266.

52. Zhong, Y.; Zhong, Y.; Zhang, Y.-P.; Liu, Y.-X. Thick branes with inner structure in mimetic gravity. Eur. Phys. J. 2018, 78, 45. [CrossRef]

53. Bazeia, D.; Ferreira, D.A.; Moreira, D.C. First order formalism for thick branes in modified gravity with Lagrange multiplier. Europhys. Lett. 2020, 129, 11004. [CrossRef]

54. Afonso, V.I.; Bazeia, D.; Losano, L. First-order formalism for bent brane. Phys. Lett. 2006, 634, 526-530. [CrossRef]

55. Janssen, B.; Smyth, P.; Riet, T.V.; Vercnocke, B. A first-order formalism for timelike and spacelike brane solutions. J. High Energy Phys. 2008, 2008, 0804. [CrossRef]

56. Menezes, R. First Order Formalism for Thick Branes in Modified Teleparallel Gravity. Phys. Rev. 2014, 89, 125007. [CrossRef]

57. Bazeia, D.; Ferreira, D.A.; Lobo, F.S.N.; Rosa, J.L. Novel modified gravity braneworld configurations with a Lagrange multiplier. arXiv 2020, arXiv:2011.06240.

58. Bazeia, D.; Gomes, A.R. Bloch brane. J. High Energy Phys. 2004, 2004, 012. [CrossRef]

59. Liu, Y.-X.; Yang, J.; Zhao, Z.-H.; Fu, C.-E.; Duan, Y.-S. Fermion Localization and Resonances on A de Sitter Thick Brane. Phys. Rev. 2009, 80, 065019. [CrossRef]

60. Cui, Z.-Q.; Lin, Z.-C.; Wan, J.-J.; Liu, Y.-X.; Zhao, L. Tensor Perturbations and Thick Branes in Higher-dimensional $f(R)$ Gravity. J. High Energy Phys. 2020, 12, 130. [CrossRef] 